\title{
INSOMNIA DISORDER: CLINICAL AND RESEARCH CHALLENGES FOR THE
}

\section{$21^{\text {ST }}$ CENTURY}

Luigi Ferini-Strambi ${ }^{1,2}$, Reto Auer ${ }^{3}$, Bjørn Bjorvatn ${ }^{4}$, Vincenza Castronovo ${ }^{2}$, Oscar Franco ${ }^{5}$, Luca Gabutti ${ }^{6}$, Andrea Galbiati ${ }^{1,2}$, Goeran Hajak ${ }^{7}$, Ramin Khatami ${ }^{8}$, Tsuyoshi Kitajima ${ }^{9}$, Doug McEvoy ${ }^{10}$, Christoph Nissen ${ }^{11}$, Michael Perlis ${ }^{12}$, Dirk A Pevernagie ${ }^{13}$, Winfried Randerath ${ }^{14}$, Dieter Riemann ${ }^{15}$, Giovanni Rizzo ${ }^{16}$, Eus Van Someren ${ }^{17}$, Alexandros Vgontzas ${ }^{18}$, Fabrizio Barazzoni ${ }^{19}$, Claudio Bassetti ${ }^{20,21}$ on behalf of the European

\author{
Sleep Foundation
}

(1) "Vita-Salute" San Raffaele University, Milan, Italy

(2) IRCCS San Raffaele Scientific Institute, Neurology - Sleep Disorders Center, Milan, Italy

(3) Institute of Primary Health Care (BIHAM), University of Bern, Bern, Switzerland

(4) Department of Global Public Health and Primary Care, University of Bergen, Norway and Norwegian Competence Center for Sleep Disorders, Haukeland University Hospital, Norway

(5) Institute of Social and Preventive Medicine, University of Bern, Bern, Switzerland

(6) Institute of Biomedicine, University of Southern Switzerland, Lugano, Switzerland

(7) Department of Psychiatry, Psychosomatic Medicine and Psychotherapy, Social Foundation Bamberg, Bamberg, Germany

(8) Barmelweid Academy, Center of Sleep Medicine, Sleep Research and Epilepsy, Klinik Barmelweid, Barmelweid, Switzerland

(9) Department of Psychiatry, Fujita Health University School of Medicine, Toyoake, Japan

(10) Adelaide Institute for Sleep Health, College of Medicine and Public Health, Flinders University, South Australia, Australia

(11) University Hospital of Psychiatry and Psychotherapy, University of Bern, Bern, Switzerland

(12) Department of Psychiatry, Behavioral Sleep Medicine Program, Perelman School of Medicine, University of Pennsylvania, Philadelphia, Pennsylvania, USA

(13) Department of Internal Medicine and Pediatrics, Ghent University, Ghent, Belgium

(14) Institute of Pneumology at the University of Cologne, Solingen, Germany

(15) Department of Psychiatry and Psychotherapy, Medical Centre - University of Freiburg, Faculty of Medicine, University of Freiburg, Germany

(16) IRCCS Istituto delle Scienze Neurologiche di Bologna, Bologna, Italy

(17) Netherlands Institute for Neuroscience, Department of Sleep and Cognition, the Netherlands; Departments of Integrative Neurophysiology and Psychiatry, Center for Neurogenomics and Cognitive Research, VU University,

Amsterdam UMC, Amsterdam Neuroscience, Amsterdam, the Netherlands

(18) Sleep Research and Treatment Center, Department of Psychiatry, Pennsylvania State University, College of Medicine, Penn State Hershey Medical Center, Hershey, PA, USA

(19) Ente Ospedaliero Cantonale (EOC) Head Office, Bellinzona, Switzerland

(20) Department of Neurology, Inselspital University Hospital, University of Bern, Bern, Switzerland

(21) Department of Neurology, Sechenov University, Moscow-Russia

This article has been accepted for publication and undergone full peer review but has not been through the copyediting, typesetting, pagination and proofreading process, which may lead to differences between this version and the Version of Record. Please cite this article as doi: 10.1111/ENE.14784

This article is protected by copyright. All rights reserved 
Word count: 8589 (Total word count of the manuscript including title page, references, and abstract)

Running title: Insomnia in 21 century

\section{Corresponding author:}

Luigi Ferini-Strambi, MD

Full Professor of Neurology

Chair, Dept. of Neurology OSR-Turro

Director, Sleep Disorder Center

Vita-Salute San Raffaele University, Milan, Italy

Tel.: +390226433363

e-mail: ferinistrambi.luigi@hsr.it

This article is protected by copyright. All rights reserved 
PROF. LUIGI FERINI STRAMBI (Orcid ID : 0000-0003-2867-5424)

DR. GIOVANNI RIZZO (Orcid ID : 0000-0002-9718-2044)

PROF. CLAUDIO L BASSETTI (Orcid ID : 0000-0002-4535-0245)

Article type : Original Article

\begin{abstract}
Background: Insomnia is a common and debilitating disorder that is frequently associated with important consequences for physical health and well-being.

Methods: An international expert group considered the current state of knowledge based on the most relevant publications in the previous 5 years, discussed the current challenges in the field of insomnia, and identified future priorities.

Results: The association of trajectories of insomnia with subsequent quality of life, health, and mortality should be investigated in large populations. Prospective health economic studies by separating the specific costs driven specifically by insomnia and costs attributable to its long-term effects are needed. Ignoring the heterogeneity of insomnia patients leads to inadequate diagnosis and inefficient treatment. Individualized interventions should be promoted. More data are needed on both the impact of sleep on overnight effects, such as emotion regulation, and the potential compensatory effort to counteract diurnal impairments. Another gap is the definition of neurocognitive deficits in insomnia patients compared to normal subjects after chronic sleep loss. There are also a number of key gaps related to insomnia treatment. Expert guidelines indicate cognitive-behavioral therapy for insomnia (CBT-I) as first line treatment. They neglect, however, the reality of major health care providers. The role of combined therapy, CBT-I plus pharmacological treatment, should more extensively evaluated.
\end{abstract}

This article is protected by copyright. All rights reserved 
Conclusion: While insomnia disorder might affect large proportions of the population, there are a number of significant gaps in the epidemiological/clinical/research studies carried out to date. In particular, the identification of different insomnia phenotypes could allow more cost-effective and efficient therapies.

\section{INTRODUCTION}

Since Hippocratic times lifestyle has constituted a cornerstone of health and prevention. The three key pillars of lifestyle are diet, activity and sleep. Sleep, a key part of our life, has been challenged by the westernization of lifestyle and by a dramatic increase in the use of technology in daily life.

Insomnia disorder is the second-most prevalent mental disorder ${ }^{1}$ and is the most common sleep complaint $^{2}$. Insomnia is defined by difficulties initiating or maintaining sleep accompanied subjectively experienced to have adverse consequences for daytime functioning, and occurring in spite of appropriate circumstances and opportunity for sleep. If the complaints occur at least three times a week and last for at least three months, the diagnostic criteria for insomnia disorder are met. Importantly, according to both International Classification of Sleep Disorder $3^{\text {rd }}$ edition ${ }^{3}$ and DSM $-5^{4}$ the distinction between primary and secondary insomnia was removed in favor of an umbrella category for insomnia disorder which can be used also when insomnia is comorbid with other conditions. This modification reflects understanding that although insomnia frequently accompanies other disorders, it can also precede the comorbid condition, persist despite effective treatment of the comorbid condition, or aggravate the symptoms of the comorbid condition ${ }^{5}$. The prevalence of acute insomnia symptoms in the general population is estimated to be up to $37 \%$ per annum $^{6-9}$. The prevalence of chronic insomnia is about $10-20 \%$ and is more prevalent in women, older adults, and individuals of lower socioeconomic status ${ }^{3,10,11}$. Although insomnia shows high prevalence, it is seldom adequately assessed. This likely reflects the fact that most medical school and internal and family medicine residency training programs dedicate very little time to the assessment and treatment of sleep disorders (in general) and to the evaluation and management of insomnia (in specific) ${ }^{12,13}$. The more recent diagnostic criteria ${ }^{3,4}$ recommend that 'insomnia disorder' should be coded "whenever diagnostic criteria are met whether or not there is a co-existing physical, mental or sleep disorder". Insomnia is ubiquitous, so it is important that clinicians and in particular, general practitioners, have the ability to identify patients with insomnia. The identification of brief, reliable and valid screening tools to evaluate insomnia disorder in everyday clinical practice is undoubtedly needed.

Several neurologic disorders are frequently complicated by comorbid insomnia. Insomnia may influence the severity of both epilepsy and headache, and its treatment may improve seizure and

This article is protected by copyright. All rights reserved 
headache frequency ${ }^{14}$. Insomnia may be a potentially modifiable risk factor for Alzheimer's disease $(A D)^{15}$. Insomnia symptoms have been reported in up to $80 \%$ of patients with Parkinson' disease (PD), but according to the diagnostic criteria of "insomnia disorder" prevalence rate is $43 \%{ }^{16}$. At least $40 \%$ of subjects affected by multiple sclerosis (MS) have chronic insomnia, and the prevalence could be higher in relation to underdiagnosis ${ }^{17}$. Moreover, insomnia is frequently associated with sleep breathing disorder. Indeed, up to $58 \%$ of patients with sleep apnea report symptoms indicative of co-morbid insomnia ${ }^{18}$.

An important aspect is to establish when the clinical assessment cannot be considered enough in the diagnostic approach. Polysomnography (PSG) and actigraphy provide estimates of objective sleep and wake duration, however, a meta-analysis showed that PSG measures in patients suffering from insomnia may not deviate from normal values in many patients and do not capture the subjective experience of being awake ${ }^{19}$.

Some studies in the last years indicated precise insomnia phenotypes. For instance, insomnia with short sleep duration (ISSD) is recognized as the most severe phenotype ${ }^{20}$. All-cause mortality, incident cardiovascular diseases (CVD), hypertension and diabetes, are higher in individuals with ISSD in comparison to other phenotypes ${ }^{21}$. It should be clarified whether genetics or neuroimaging may offer a concrete contribution in the definition of different phenotypes of insomnia.

Lastly, insomnia is a treatable disorder. There is a very substantial level 1 evidence-base, evaluating pharmacological treatments and cognitive-behavioural therapy for insomnia (CBT-I). Expert guidelines recommend CBT-I as first line treatment for insomnia, but it should be recognized that inperson CBT-I may be difficult to access. Accordingly, dissemination of information regarding assessment and access to referral information is critical.

The present overview results from a collaborative effort of a group of international experts in the fields of interest. It summarizes the most important recent research and medical findings in the field of insomnia, and identifies the unsolved needs.

\section{METHODS}

This paper summarizes the results of a symposium (Think Tank 2019) organized by the European Sleep Foundation, following a standardized approach used in three previous editions of Think Tank $^{22-24}$. The original idea of these meetings was conceived by some members of Sleep-Wake Disorders Scientific Panel of the European Academy of Neurology. Thirty international experts were invited to Think Tank 2019 and 23 participated to the conference, held from 18 to 20 October 2019 in Baveno, Italy. Experts were from the domains of sleep research, sleep medicine, neurology,

This article is protected by copyright. All rights reserved 
psychiatry, family medicine and public health. Ten countries from four continents, Europe, US, Japan and Australia were represented to reflect different healthcare systems. Five major topic areas were used to guide the definition of important clinical questions and challenges regarding insomnia including: 1) Epidemiology and public health, 2) The role of the family medicine and the sleep medicine center in the assessment and treatment, 3) Phenotype heterogeneity, 4) Neurocognitive performance, and 5) Non-pharmacological and pharmacological treatments.

The participating experts were divided into five subgroups. Each subgroup defined the most important clinical challenges and research priorities by discussing the most relevant papers published in the last five years for each of the five major topic areas. These groups drafted five documents that were then revised by the conference leaders.

\section{RESULTS / CONSENSUS STATEMENTS}

\section{Session 1 - Public Health and Insomnia}

Currently, the prevalence of short sleep duration and acute insomnia symptoms in the general population are approximately $29 \%$ and $37 \%$ respectively ${ }^{6-9,25}$. Of these, approximately two thirds can be considered poor sleepers while the remaining require clinical support and treatment and can be considered as suffering from chronic insomnia disorder. Persons with poor sleep have a higher chance of developing insomnia disorder ${ }^{26}$. The prevalence of chronic insomnia is about $10-20 \%$ and is more prevalent in women, older adults and individuals of lower socioeconomic status ${ }^{3}$. Additional factors related to poor sleep and/or chronic insomnia include life event stress, physical illness and injury, response to sleeplessness, and to some extent diet (caffeine, alcohol, and energy drinks), iatrogenic effects from medications, substance use and abuse, electronic device use, socioeconomic status, occupational status, and environmental noise pollution (including light and sound).

Over the last 8 decades, there has been an exponential rise in the number of papers on insomnia, reflecting increased attention to the epidemiology of insomnia, theory regarding the etiology and pathophysiology of insomnia, assessment and classification, treatment development, and clinical trials of medical and behavioral therapeutics for insomnia (See Figure 1).

This article is protected by copyright. All rights reserved 


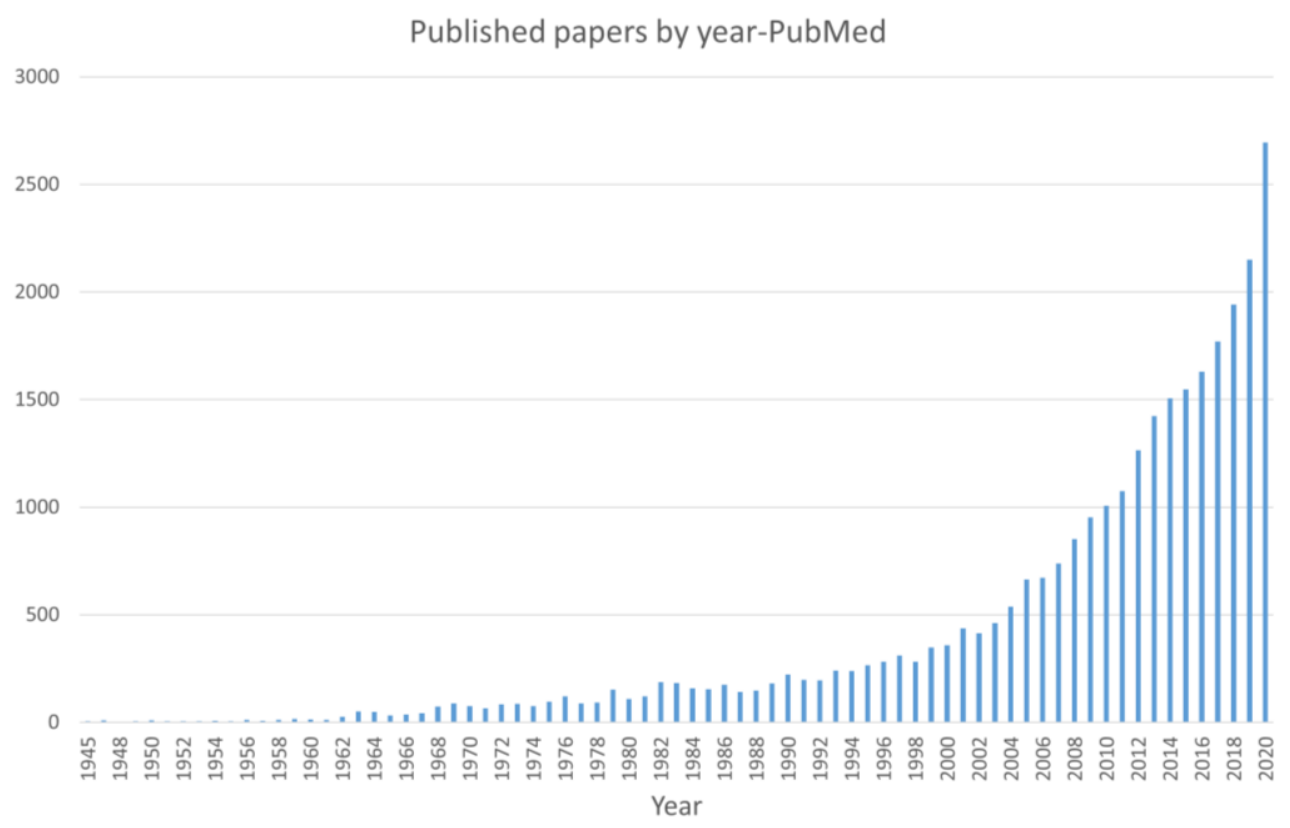

Figure 1. Number of published papers by year for Insomnia as the Major MeSH topic.

One particularly productive strategy has been the evaluation of the consequences of one particular insomnia phenotype: Insomnia with Short Sleep Duration (ISSD). Recent studies show that ISSD has a differential response to treatment based on this phenotypic distinction ${ }^{27}$. Besides the prognostic impact of phenotypes, the impact of insomnia severity has been analyzed by the number of complaints and its associated risk. Longitudinal data support a dose-dependent association of baseline number of insomnia symptoms and the risk of heart failure ${ }^{28}$. In addition, the presence of insomnia in patients with established heart failure is an independent predictor of cardiac events ${ }^{29}$. Individual insomnia symptoms also have prognostic value. A meta-analysis of 23 cohorts estimated the risk of having difficulties falling asleep, having difficulties maintaining sleep, early morning awakening and non-restorative sleep. Both difficulties falling and maintaining sleep were associated with an increased risk of CVD events ${ }^{30,31}$.

On the other hand, sufficient sleep duration in addition to all four traditional healthy lifestyle factors (physical activity, nutrition, smoking and alcohol intake) was associated with 65\% lower risk of CVD and $83 \%$ lower risk of fatal CVD in one large European cohort $^{32}$.

However, it is still unknown whether the association of insomnia with cardio-metabolic risk is causal due to reverse causation bias and residual confounding factors inherent to observational studies. Furthermore, how sleep is measured and the mechanisms explaining the association of sleep disorders with health issues and mortality remain unclear. Therefore, recent and ongoing research are addressing the need to integrate other lifestyle factors as possible mediators in the association

This article is protected by copyright. All rights reserved 
of insomnia and CVD risk. Remarkably, individuals with high Mediterranean diet score (MDS) are less likely to have ISSD compared with those with low $\mathrm{MDS}^{33}$.

\section{Future priorities and next steps include:}

1. To further investigate in large populations with heterogeneous characteristics the association of trajectories of insomnia with subsequent health, quality of life and mortality.

2. To perform prospective health economic studies separating the costs driven specifically from insomnia and costs attributable to its long-term effects.

3. To use large-scale datasets from consumer apps and wearables to better understand the associations of the continuum of physical activity, diet, stress and sleep together with other lifestyle factors, with health, quality of life and mortality.

4. To assess the causal pathways between insomnia and sleep disorders and risk factors (e.g. hypertension, glucose metabolism), diseases (e.g. affective disorders, neurodegenerative conditions, cardiovascular disease) and mortality. Analyses of prospective cohort studies applying rigorous statistical methods enabling causal inference, in particular Mendelian randomisation are promising approaches.

5. To design and implement public health policies and strategies aimed to improve sleep health in the population (e.g. noise reduction, flexible working conditions, improvements in physical activity) as a mean to improve population and planetary health.

\section{Session 2 - Insomnia Disorder Diagnosis: The Role of The Family Medicine.}

Internal and family medicine practitioners, who are at the forefront of health care delivery in most European countries and many others worldwide, are ideally placed to diagnose and manage insomnia. There are few studies on insomnia among patients visiting their general practitioner $(G P)^{34}$. Sleep complaints often co-occur with other existing psychological and somatic conditions, thus the prevalence of insomnia is likely to be higher in patients visiting GPs in comparison to the prevalence found in the general population. In what is perhaps the first study of this issue, some 20 years ago, the prevalence of insomnia was reported to be over $50 \%$ in primary care patients ${ }^{35}$. A more recent study in consecutive patients approached in the GP's waiting room in New Zealand showed a prevalence of insomnia of $41 \%^{36}$. Another study in consecutive and unselected patients visiting their GPs in Norway found a prevalence of $53.6 \%{ }^{37}$. Are GPs aware of how common insomnia may be among the patients visiting their practice? Interestingly in another Norwegian study, GPs estimated the prevalence of sleep problems among their patients to be $11 \%^{38}$. GP is usually the first point of contact, and a survey indicated that $79 \%$ of GPs see subjects with a sleep complaint at least once a week ${ }^{39}$. Figure 2 highlights the importance of considering primary care practitioners in the

This article is protected by copyright. All rights reserved 
diagnosis and treatment of chronic insomnia. The "Ecology of Medical Care," 40 has provided a framework for thinking about the organization of health care, medical education, and research. If we consider a population of 1000 adults, of which half would experience insomnia symptoms per year and $15 \%$ chronic insomnia symptoms, the majority will be seen in family medicine and community settings and a minority in specialized sleep centers. Population-level chronic insomnia treatment policies should integrate family medicine and foster interdisciplinary collaboration with specialized sleep centers.

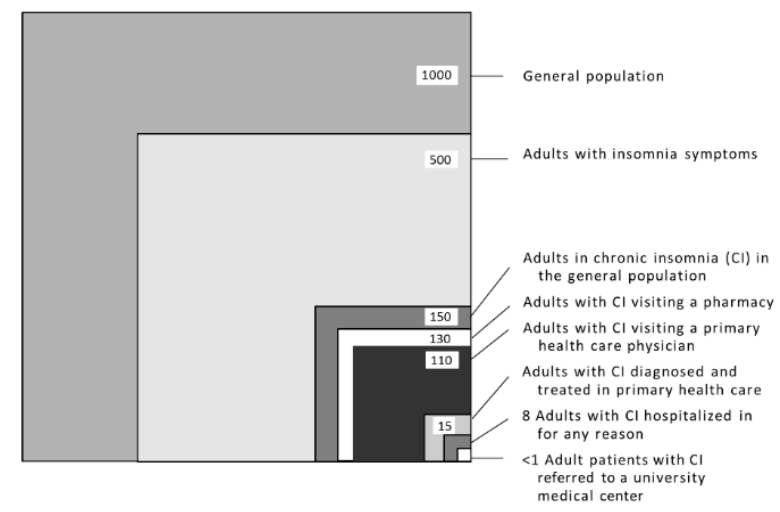

Figure 2. The ecology of healthcare revisited for chronic insomnia. Estimates of healthcare utilization per year of a population of 1000. Each box represents a subgroup of the largest box, which comprises 1000 persons. Data are for adult persons of all age.

However, the evidence points to a failure of family care practitioners to enquire about sleep difficulties and a reluctance for insomnia sufferers to seek their advice. This "don't ask, don't tell" phenomenon is not well-understood but likely arises from a lack of knowledge on the part of both patients and GPs concerning the adverse health consequences of chronic insomnia. Despite GPs believing that a sleep assessment and the identification of insomnia are crucial, the time to devote to each patient is limited. Thus, a brief measure for insomnia screening is important. The two-item Sleep Condition Indicator may help GPs to rapidly screen insomnia in the clinical practice ${ }^{41,42}$ (See table 1). An even shorter assessment can be made with the UK Biobank multiple choice question "Do you have trouble falling asleep at night or do you wake up in the middle of the night?". The answer "usually" distinguishes diagnosed people with insomnia from controls answering "sometimes" or "never/rarely", with an accuracy of $0.91^{43}$.

\section{Score}

$\begin{array}{lllll}4 & 3 & 2 & 1 & 0\end{array}$

Thinking about the past month, to what extent has poor sleep troubled you in general?

Not at all A little $\quad$ Somewhat Much $\quad$ Very much

This article is protected by copyright. All rights reserved 
Thinking about a typical night in the last month, how many nights a week do you have a problem with your sleep?

$0-1$

2

3

4

5-7

Table 1. Scoring instructions: Add the item scores to obtain the Sleep Condition Indicator (SCI) total (minimum 0, maximum 8). A higher score means better sleep ${ }^{34}$. Scores $\leq 2$ indicate bad sleep quality.

Concerning the management of insomnia by GPs, there is limited evidence. Sleep hygiene education (SHE) is the most commonly used non pharmacological treatment in general practice ${ }^{44}$. A recent systematic review and meta-analysis showed that SHE is substantially less efficacious than other non-pharmacological treatments, such as insomnia-specific cognitive behavioral therapy (CBT-I) ${ }^{45}$. CBT-I is a multimodal treatment that includes SHE but has as the putatively more active components, sleep restriction and stimulus control therapies. Some practitioners also augment standard CBT-I with one or more of the following: more rigorous forms of cognitive therapy, mindfulness training, behavioral experiments, and/or bright light therapy. The data on the effectiveness and safety of CBT-I is strong and CBT-I is now considered the first line therapy for chronic insomnia in major guidelines throughout the world ${ }^{11,46}$. This said, of the few family medicine practitioners who have heard of CBT-I, they believe it to be outside their scope of practice and find it difficult to refer patients. Potential solutions to this problem are 1) disseminate information about provider directories (e.g., https://cbti.directory/), 2) consider access to CBT-I via specialists who provide telehealth care, and 3) consider prescribing accessing on-line CBT-I programs (e.g.,https://sleepful.me/ https://www.somryst.com/; https://www.sleepio.com/). With respect to the last of these options, use of the on-line programs may require that the individual health care system have a contract with the on-line provider and/or that the treatment be part of the system's formulary. Finally, the various on-line programs may work best when a health provider concurrently monitors patient progress for treatment response or failure.

Finally, patients who fail to articulate their problem to their family physician self-medicate with ineffective or even harmful agents (e.g. alcohol); and the family physician who is made aware of the patient's problem, prescribes hypnotic drugs or off-label sleep promoting medications, despite knowing of their potential long term risks.

\section{Future priorities and next steps include:}

1. To inform the general community and family health professionals of

a. The serious personal, societal and medical costs of chronic insomnia, and that

b. Effective non-pharmacological treatment such as CBT-I is available

This article is protected by copyright. All rights reserved 
c. The potential harms and addictive potential of drug therapies such as benzodiazepines and z-drugs when used to treat chronic insomnia.

2. To provide to family medicine practitioners with a chronic insomnia "toolkit" ** enabling

a. screening for chronic insomnia with simple questions to quickly identify the high risk patient, who then

b. diagnosis of chronic insomnia and other sleep disorders with more detailed, yet brief, clinical sleep assessment

c. shared decision making between clinicians and patients with patient decision aids helping patients choose the treatment options that matches their preferences and values. The decision aid would highlight the benefits, risks and treatment burdens of non-pharmacological and pharmacological therapies (CBT-I).

d. interdisciplinary care of chronic insomnia with algorithms presenting when to refer to specialized sleep health care

3. To create interdisciplinary networks between family medicine practitioners and sleep center specialists to
a. Provide the necessary CBT-I training
b. Take referrals for complex or treatment-resistant cases

\section{Session 3 - Insomnia and Phenotype Heterogeneity}

In contrast to what the name suggests, insomnia differs strongly from curtailed sleep duration in otherwise good sleepers. Polysomnography recordings show that people with insomnia disorder do sleep, but in a fragmented and instable way ${ }^{19}$.

Insomnia complaints commonly co-occur with somatic-, mental- and other sleep disorders and usually worsen their severity. Clinicians should especially be aware of the common co-occurrence of insomnia with other sleep disorders ${ }^{47-49}$. It is only very seldom appropriate or helpful to regard insomnia as 'secondary', as merely a symptom of another disorder. On the contrary, as for other disorders ${ }^{50}$, in a better conceptualization insomnia is actionable and successful intervention can ameliorate the severity of other symptoms.

A continuing question is whether insomnia is a single homogeneous disorder. Heterogeneity of complaints and treatment response suggested the existence of different types and/or 'subtypes' with different underlying mechanisms. In terms of presenting complaints, several subtypes are clearly apparent, classically referred to as: initial, middle, late, and mixed insomnia. Clinically, there are 3-4 presentations that have been classified in previous versions of the DSM and ICSD

This article is protected by copyright. All rights reserved 
nosologies ${ }^{3,4}$, including: Psychophysiologic Insomnia; Paradoxical Insomnia (i.e., Sleep State Misperception Insomnia); Idiopathic Insomnia; and possibly Physiologic Insomnia. While these types are prototypic, the degree to which they are orthogonal and/or reliably diagnosed have led some to simply adopt the global classification of "Insomnia Disorder"51,52. This does not mean that insomnia is a homogeneous disorder, on the contrary. It means that top-down subtype definition may not necessarily result in robust subtyping.

Converging support suggests a separate subtype of people with insomnia that sleep less than 6 hours, as assessed either during the first night of polysomnography, or on average across multiple nights $^{20}$. The ISSD subtype is in addition characterized by a longer history of insomnia, more deviant biomarkers, increased health risks, and treatment resistance ${ }^{27,53}$. All mentioned subtypes have a limited consistency over time ${ }^{52}$, e.g. of the people diagnosed with ISSD after one night, two third may not fulfill the criterion anymore at a second night ${ }^{53}$ More recently, big data and data-driven methods have revealed other robust and replicable subtypes based on multivariate profiles of traits and personal history and linked to biological features ${ }^{54}$.

We expect that these novel subtyping efforts will increase the chances to reveal distinguishable underlying mechanisms leading to seemingly similar sleep complaints, but possibly requiring different treatments. An unmet current need for big data subtyping is to assess individual differences in responses to different treatments. Doing so will ultimately allow us to predict which treatment works best for whom.

Genome wide associations studies (GWAS) have recently addressed insomnia risk genes. In fact, across all disorders, the largest GWAS to date ( $\mathrm{N}=1,3$ million) for any trait or disorder specifically addressed insomnia ${ }^{55}$. Findings indicate that insomnia is a highly polygenic complex trait. As commonly found in GWAS, there is considerable pleiotropy and overlap of risk genes for insomnia and other phenotypes and disorders. Interestingly, the overlap is stronger with mood and anxiety phenotypes than with sleep- related phenotypes. Also clock-related genes are not particularly represented. The use of GWAS goes beyond just finding genes that each explain very little variance. The availability of gene-expression databases has made it possible to suggest particular brain areas and cell types most likely to convey the genetic risk that multiple gene variants convey. Concertedly, identified brain areas, cell types and phenotypic and genotypic associations provide little support for insomnia being a disorder of sleep regulation and rather suggest it to concern problematic regulation of stress and emotion.

\section{Future priorities and next steps}

This article is protected by copyright. All rights reserved 
To move forward in understanding insomnia, we would ideally capitalize on existing large cohort studies with extensive multivariate biomarkers. If cohorts have not assessed insomnia yet, we advocate the inclusion of at least a single question indicator variable of the diagnosis of insomnia. The UK Biobank question of insomnia for example shows good accuracy as compared to a diagnosis based on a structured interview ${ }^{43}$.

Next steps include:

1. To use data-driven approaches in existing databases to find novel sleep-EEG and actigraphy features of relevance for insomnia

2. To monitor the release of new wearable (e.g. oxygen desaturation + actigraphy) and evaluate their use to find features of relevance for insomnia

3. To use advanced multivariate methods like symptom network analysis to disentangle the risk of morbidity and mortality insomnia conveys relative to common comorbidity and symptoms

4. To commence a collaborative intervention-trials registry for assessment of individual profiles treatment outcomes and use data driven (e.g. machine learning) approaches to ultimately predict who responds best to which treatment.

\section{Session 4- Neurocognitive Performances in Insomnia Disorder}

Although insomnia is commonly conceived as a nighttime disorder, its complaints and consequences extend well beyond the nocturnal period. Indeed, its pathophysiology has been predominantly framed on the hyperarousal mode ${ }^{56}$. According to this view, that is based on both clinical observation and scientific evidence, insomnia is characterized by an augmented state of arousal that is expressed on autonomous nervous system, behavioral, cognitive-emotional and cortical level throughout the entire day. In spite of this hyperactivation, patients frequently report impaired quality of life and a variety of adverse health conditions, such as depression, and there is also evidence that insomnia represents a risk factor for several others psychopathologies such as anxiety, alcohol abuse and psychosis ${ }^{57}$. Insomnia is also associated with increased absenteeism at work and loss of productivity probably due to an impaired diurnal functioning. Accordingly, patients complain of decreased cognitive functioning that frequently involve memory, executive functions, decision making and, more in general, work related mistakes. Notably, these impairments are not always corroborated by objective investigations. A meta-analysis reported that insomnia had mild to moderate impairments on specific cognitive domains. Specifically, patients performed poorly on tasks assessing working memory, episodic memory and problem solving and they exhibited only mild to moderate impairment for several attentional processes. However, their performances did not differ in comparison to good sleepers for psychomotor processes, verbal functions, procedural

This article is protected by copyright. All rights reserved 
memory and global cognitive functioning ${ }^{58}$. Some have argued that the observed lack of objective findings with respect to daytime impairment belies the actual or core complaint of those with insomnia; it is not so much a matter of successful output/performance, it is the effort required to reach these ends that seems affected by poor sleep continuity and/or reduced sleep duration ${ }^{59}$.

Another relevant issue is represented by the discrepancies between subjective and objective sleep assessment. Large data sets analysis reported only minor polysomnographic alterations. In particular, results showed that patients with insomnia have a sleep continuity disruption and a reduction of slow wave sleep (SWS) and rapid eye movement sleep compared to good sleepers ${ }^{19}$. However, it is recognized in the scientific community that insomnia patients are prone to underestimate their total sleep time and overestimate their sleep onset latency and wakefulness after sleep onset. This implies that sleep misperception might be a core feature and an essential aspect to understand the disorder but unveil also a chicken-and-egg problem: sleep state misperception is cause or effect of insomnia? Interestingly, there is also evidence that local aspects of both sleep and wakefulness might help in understanding and solving the problem ${ }^{60,61}$.

\section{Future priorities and next steps include:}

1. To investigate brain mechanisms of poor subjective sleep quality by:

a. Challenging the hyperarousal concept

b. Cycling Alternating Pattern (CAP)

c. Slow Wave Sleep and homeostasis

d. Concepts of local wakefulness and sleep

e. Concepts of orchestration of brain oscillations (e.g. spindle activity phase-locked to) sleep slow oscillations

f. Provocation tests (e.g. homeostatic response to sleep restriction, probe individual sleep need)

2. To investigate the mechanisms of subjective daytime impairments and neurocognitive performance deficits by:

a. further assessment of sleep impact on overnight effects, such as emotion regulation

b. the assessment of potential compensatory effort to counteract diurnal impairments

c. the assessment of neurocognitive deficits in insomnia patients versus the effect of chronic sleep restriction in normal subjects

3. To investigate the potential causality of the links between insomnia and adverse health conditions, such as dementia.

\section{Session 5- Treatment of Insomnia Disorder}

This article is protected by copyright. All rights reserved 
In recent years, evidence has emerged that patients with insomnia face a tremendous chance to be effectively treated in the short and in the long term ${ }^{62}$. In general, pharmacological agents have been tested in short-term studies and there is little evidence for their effectiveness or safety beyond 3-4 month treatment ${ }^{63}$. The robust efficacy of CBT-I has been unequivocally proven in the short and long term ${ }^{64,65}$. Respective expert guidelines which provide CBT-I as first line treatment can rely on a valid database ${ }^{11,46}$. However, they neglect the reality of major health care providers. The de facto insomnia treatment is provided by GPs. GPs claim that CBT-I transfer into wide clinical use requires more detailed data on the way and quality of its application in primary care settings before being recommended for the broad use ${ }^{66}$. Patients as well as doctors education and awareness programs promise a better use of CBT-I and its implementation. However, this requires a scientific approach to validate their quality and efficiency. The significant mismatch between expert recommendations and availability of CBT-I treatment calls for, as noted above, a broader conceptualization about how to inform primary care practitioners regarding how to access / refer for CBT-I (see above, Section 2).

A recent study showed that CBT-I is feasible in patients with MS and produces significant improvements in insomnia severity, sleep quality, as well as in fatigue and depression symptoms ${ }^{17}$. Moreover, small studies showed that CBT-I is safe and efficacious treatment also in PD, but strategies to enhance therapy adherence are needed ${ }^{16}$. Strikingly, CBT-I is also effective in improving insomnia symptoms in patients with untreated obstructive sleep apnea and increase subsequent continuous positive airway pressure therapy outcomes ${ }^{67}$.

Concerning pharmacological treatment, an increasing number of field studies as well as data from controlled studies have revealed a positive correlation of the use of benzodiazepine receptor agonistic hypnotics (BRZA) with medication abuse and dependence, infection rates, falls, accidents, and mortality ${ }^{68,69}$. The arising criticism on sleeping pills has reduced prescription rates of BRZA world-wide. This reaction has taken place in spite of the fact that most long-term field studies lack the reliable proof that hypnotic intake is causing harm, rather than an epiphenomenon of a genuine sick population with comorbid or coincident insomnia. On the other hand, it has been recently observed that one in five new users of sedative-hypnotics will become a long-term user, but only $0.5 \%$ will become excessive users ${ }^{70}$. A switch to the prescription of sleep promoting drugs of other pharmacological classes is observed in most industrialized countries. Sedating antidepressant, some herbal drugs, orexin receptor antagonists, and antipsychotics have short-term efficacy in insomnia patients ${ }^{11,62,71}$. The scientific community, however, is asked to assess their benefit/risk ratio especially in the long term. In particular, serious cardiac and metabolic effects of some agents challenge their off label use in chronic insomnia. Moreover, a recent systematic review on drug treatments for insomnia people with dementia showed that there was evidence of some beneficial

This article is protected by copyright. All rights reserved 
effects from trazodone and orexin antagonists but larger long-term trials are needed also for the assessment of adverse effects ${ }^{72}$. The authors found no evidence for beneficial effects of melatonin (up to $10 \mathrm{mg}$ ) or ramelteon, a melatonin receptor agonist. However, in another recent review on the management of insomnia in PD, the authors suggested the use of prolonged release (PR) melatonin or doxepine for patients aged $>65$ years, and PR melatonin or doxepine or eszopiclone or zolpidem for patients aged $<65$ years ${ }^{16}$. Moreover, the authors reported that there are few safety data concerning the long-term use of sedative-hypnotic drugs also in $\mathrm{PD}^{16}$.

Currently, and in the future, it will be insufficient for pharmaceutical companies to convince consumers that a particular drug provides significant sleep improvement. Rather they must provide unequivocal evidence that its benefit in health improvement outweighs its potential risk and harm. It also appears to be a serious demand to further investigate the mechanisms of action in order to better understand the heterogeneity of these drug classes.

Clinical experience in daily practice has created the wording that "getting to sleep with drugs is just a question of dosing". Limitations of this approach arise mainly from unwanted side effects such as dose- and drug-dependent hangover effects and lack of improvement of daytime well-being and functioning with increasing treatment dose. It rarely drives doctors to a general critical position towards the use of sleeping pills. Unfortunately, the current debate on the benefit-risk ratio of hypnotics is rather emotional and mainly driven by the concern for abuse and dependence. No issue of hypnotic use has gained more attention during the last decades. It is true that some long-term studies with medication intake on a nightly basis over up to one year have failed to show a cure of the disease after end of treatment. It is, however, also true that dose escalation has rarely been found. Furthermore, withdrawal of modern hypnotic agents such as selective benzodiazepine receptor agonists, melatonin agonists, sleep promoting antidepressants, or orexin-receptor antagonists do not exhibit similar withdrawal symptoms or rebound insomnia even after abrupt cessation of treatment as older types of hypnotics. This does not rule out psychological dependence to stimulate patients regaining treatment to restore their former satisfaction with the drug-related sleep benefit.

Following the widely accepted and evidence based hypothesis that a chronic neuronal hyperarousal accounts for chronic insomnia, a continuous arousal-suppressing medication could be seen as an appropriate drug class to be evaluated for treatment. Physicians in general have accepted that patients with diabetes or arterial hypertension take their medication on a regular basis. Moreover, patients with these diagnoses have been undoubtedly shown to profit from behavioral treatments such as diet or physical activity. To prevent them from receiving a medication, however, would be

This article is protected by copyright. All rights reserved 
understood as medical misconduct Instead of arguing on the best treatment modality for patients between healthcare providers, our time and energy as healthcare professionals should go to inform patients on the menu of treatment options available, both behavioral and pharmacologic - to highlight their benefits, risks and treatment burdens. Shared decision making, with patient decision aids enabling healthcare providers to diagnose patient's preferences and values for various treatment modalities could be a method to achieve this goal.

This is even more the case when taking into account epidemiological data showing that the majority of patients suffer from acute and transient insomnia, and even among patients with chronic insomnia, sleep problems often occur only during some nights of the week. This suggests that appropriate treatment regimens rather than a fundamental pro and con debate should lead clinical decisions. Combination of CBT and intermittent, as needed, hypnotic use has been shown to improve sleep significantly while drug intake remained low or even declined over time ${ }^{73}$.

\section{Future priorities and next steps:}

1. Observational studies are required to prove CBT-I in real life settings.

2. Strategies are required to enhance CBT-I adherence in patients with PD and MS.

3. The implementation of CBT-I in neuropsychiatric disorder and in patients with comorbid obstructive sleep apnea is needed.

4. Stepped care approaches should consider the use of single CBT-I components reaching to complex programs and those with combination with pharmacological treatment.

5. Should CBT-I or hypnotic drugs be used in acute/short-term insomnia to prevent chronicity of the disorder (such as in pain treatment)?

6. Clinical studies with non-nightly drug intake with or without behavioral treatment components could clarify the efficiency of these treatment schedules in patients with transient insomnia or chronic insomniacs with not-every night sleep complaint.

7. Urgent need for studies of widely used drugs without scientific evidence (e.g. sedating antidepressants, antipsychotics, antiepileptics).

8. Long-term clinical trials for insomnia especially in neurological patients, with the assessment of adverse effects, are needed.

9. Develop patient decision aids presenting the treatment options available to patients in order to enable healthcare professionals to diagnose patient's preferences and values and move towards shared decision making.

10. Research in the future will have to question the validity of standard electrophysiological measurements in their value to assess the effect of treatments. Functional magnetic resonance

This article is protected by copyright. All rights reserved 
imaging or positron emission tomography, near infrared spectroscopy, or electroencephalographic fine structure analysis could provide a better understanding of sleep related states of consciousness, as well as of treatment effects.

\section{CONCLUSION}

Insomnia is a common and debilitating disorder that is frequently associated with important consequences for physical health and well-being. There are a number of significant gaps in the epidemiological/clinical/research studies carried out to date. From the epidemiological point of view, the association of trajectories of insomnia with subsequent health, quality of life and mortality should be investigate in large populations with heterogeneous characteristics. How sleep is measured and the mechanisms explaining the association of sleep disorders with health issues and mortality remain unclear. Moreover, prospective health economic studies by separating the specific costs driven specifically from insomnia and costs attributable to its long-term effects are needed.

Despite the high prevalence of insomnia in the primary care setting, only a small proportion of patients report sleep problems to their physician. GPs believe that a sleep assessment and the identification of insomnia are crucial, but the time to devote to each patient is limited. Thus, a brief measure for insomnia screening is important for possible referral to sleep specialist.

While insomnia disorders might affect large proportions of the population, these tend to present differently and respond differently to treatment, highlighting heterogeneous phenotypes within the insomnia spectrum. Ignoring the heterogeneity leads to inadequate diagnosis and inefficient treatment with subsequent challenges in the understanding of the causative physio-pathological mechanisms. At the same time, introducing too many phenotypes could also jeopardize diagnosis, treatment and ethological research efforts. Is there a single insomnia phenotype or multiple phenotypes, how many and how should they be defined remain fundamental issues in sleep research. However, individualized interventions should be promoted: individual recommendations of sleep duration should be based on individual sleep need (the popular notion of "8 hours for everybody" should be abandoned).

We need more data on both the impact of sleep on overnight effects, such as emotion regulation, and the potential compensatory effort to counteract diurnal impairments. Another gap is the definition of neurocognitive deficits in insomnia patients compared to normal subjects after chronic sleep restriction. There are also a number of key gaps related to insomnia treatment. Expert guidelines which provide CBT as first line treatment can rely on a valid database. They neglect, however, the reality of major health care providers. The de facto treatment is provided by general

This article is protected by copyright. All rights reserved 
care physicians, not by scientific or clinical experts in either sleep or psychotherapy. In acute short term insomnia there is a conflict whether CBT or pharmacological treatment should be used, both promising to prevent chronicity of the disorder. Combined therapy might be a solution for subchronic insomnia, but has not been proven.

\section{DATA AVAILABILITY STATEMENT}

Data sharing not applicable to this article as no datasets were generated or analysed during the current study

\section{Bibliography}

1. Wittchen HU, Jacobi F, Rehm J, et al. The size and burden of mental disorders and other disorders of the brain in Europe 2010. Eur Neuropsychopharmacol. 2011;21(9):655-679. doi:10.1016/j.euroneuro.2011.07.018

2. Mai E, Buysse DJ. Insomnia: Prevalence, Impact, Pathogenesis, Differential Diagnosis, and Evaluation. Sleep Med Clin. Published online 2008. doi:10.1016/j.jsmc.2008.02.001

3. American Academy of Sleep Medicine. The International Classification of Sleep Disorders (ICSD-3).; 2014.

4. Association AP. American Psychiatric Association, 2013. Diagnostic and Statistical Manual of Mental Disorders (5th Ed.).; 2013.

5. Riemann D, Nissen C, Palagini L, Otte A, Perlis ML, Spiegelhalder K. The neurobiology, investigation, and treatment of chronic insomnia. Lancet Neurol. Published online 2015. doi:10.1016/S1474-4422(15)00021-6

6. Perlis ML, Vargas I, Ellis JG, et al. The natural history of Insomnia: The incidence of acute insomnia and subsequent progression to chronic insomnia or recovery in good sleeper subjects. Sleep. Published online 2020. doi:10.1093/sleep/zsz299

7. Ellis JG, Perlis ML, Neale LF, Espie CA, Bastien CH. The natural history of insomnia: Focus on prevalence and incidence of acute insomnia. J Psychiatr Res. Published online 2012. doi:10.1016/j.jpsychires.2012.07.001

8. Gureje O, Oladeji BD, Abiona T, Makanjuola V, Esan O. The natural history of insomnia in the Ibadan study of ageing. Sleep. Published online 2011. doi:10.5665/SLEEP.1138

This article is protected by copyright. All rights reserved 
9. LeBlanc M, Mérette C, Savard J, Ivers H, Baillargeon L, Morin CM. Incidence and risk factors of insomnia in a population-based sample. Sleep. Published online 2009.

doi:10.1093/sleep/32.8.1027

10. Baglioni C, Battagliese G, Feige B, et al. Insomnia as a predictor of depression: A meta-analytic evaluation of longitudinal epidemiological studies. J Affect Disord. 2011;135(1-3):10-19. doi:10.1016/j.jad.2011.01.011

11. Riemann D, Baglioni C, Bassetti C, et al. European guideline for the diagnosis and treatment of insomnia. J Sleep Res. Published online 2017. doi:10.1111/jsr.12594

12. Mindell JA, Bartle A, Wahab NA, et al. Sleep education in medical school curriculum: A glimpse across countries. Sleep Med. Published online 2011. doi:10.1016/j.sleep.2011.07.001

13. Rosen RC, Rosekind M, Rosevear C, Cole WE, Dement WC. Physician education in sleep and sleep disorders: A national survey of U.S. medical schools. Sleep. Published online 1993. doi:10.1093/sleep/16.3.249

14. Ju YES, Videnovic A, Vaughn B V. Comorbid Sleep Disturbances in Neurologic Disorders. Contin Lifelong Learn Neurol. Published online 2017. doi:10.1212/CON.0000000000000501

15. Ferini-Strambi L, Galbiati A, Casoni F, Salsone M. Therapy for Insomnia and Circadian Rhythm Disorder in Alzheimer Disease. Curr Treat Options Neurol. Published online 2020. doi:10.1007/s11940-020-0612-z

16. Wallace DM, Wohlgemuth WK, Trotti LM, et al. Practical Evaluation and Management of Insomnia in Parkinson's Disease: A Review. Mov Disord Clin Pract. Published online 2020. doi:10.1002/mdc3.12899

17. Siengsukon $\mathrm{CF}$, Alshehri M, Williams C, Drerup M, Lynch S. Feasibility and treatment effect of cognitive behavioral therapy for insomnia in individuals with multiple sclerosis: A pilot randomized controlled trial. Mult Scler Relat Disord. Published online 2020. doi:10.1016/j.msard.2020.101958

18. Sweetman AM, Lack LC, Catcheside PG, et al. Developing a successful treatment for comorbid insomnia and sleep apnoea. Sleep Med Rev. Published online 2017. doi:10.1016/j.smrv.2016.04.004

19. Baglioni C, Regen W, Teghen A, et al. Sleep changes in the disorder of insomnia: a metaanalysis of polysomnographic studies. Sleep Med Rev. Published online 2014. doi:10.1016/j.smrv.2013.04.001

This article is protected by copyright. All rights reserved 
20. Vgontzas AN, Fernandez-Mendoza J, Liao D, Bixler EO. Insomnia with objective short sleep duration: The most biologically severe phenotype of the disorder. Sleep Med Rev. Published online 2013. doi:10.1016/j.smrv.2012.09.005

21. Bertisch SM, Pollock BD, Mittleman MA, et al. Insomnia with objective short sleep duration and risk of incident cardiovascular disease and all-cause mortality: Sleep Heart Health Study. Sleep. Published online 2018. doi:10.1093/sleep/zsy047

22. Bassetti CL, Ferini-Strambi L, Brown S, et al. Neurology and psychiatry: Waking up to opportunities of sleep. : State of the art and clinical/research priorities for the next decade. Eur J Neurol. Published online 2015. doi:10.1111/ene.12781

23. Bassetti CLA, Adamantidis A, Burdakov D, et al. Narcolepsy - clinical spectrum, aetiopathophysiology, diagnosis and treatment. Nat Rev Neurol. Published online 2019. doi:10.1038/s41582-019-0226-9

24. Randerath W, Bassetti CL, Bonsignore MR, et al. Challenges and perspectives in obstructive sleep apnoea. Eur Respir J. Published online 2018. doi:10.1183/13993003.02616-2017

25. St-Onge MP, Grandner MA, Brown D, et al. Sleep Duration and Quality: Impact on Lifestyle Behaviors and Cardiometabolic Health: A Scientific Statement from the American Heart Association. Circulation. Published online 2016. doi:10.1161/CIR.0000000000000444

26. Fernandez-Mendoza J, Vgontzas AN, Bixler EO, et al. Clinical and Polysomnographic Predictors of the Natural History of Poor Sleep in the General Population. Sleep. Published online 2012. doi:10.5665/sleep.1832

27. Bathgate CJ, Edinger JD, Krystal AD. Insomnia patients with objective short sleep duration have a blunted response to cognitive behavioral therapy for Insomnia. Sleep. Published online 2017. doi:10.1093/sleep/zsw012

28. Laugsand LE, Strand LB, Platou C, Vatten LJ, Janszky I. Insomnia and the risk of incident heart failure: A population study. Eur Heart J. Published online 2014. doi:10.1093/eurheartj/eht019

29. Kanno Y, Yoshihisa A, Watanabe S, et al. Prognostic significance of insomnia in heart failure. Circ J. Published online 2016. doi:10.1253/circj.CJ-16-0205

30. He $Q$, Zhang P, Li G, Dai H, Shi J. The association between insomnia symptoms and risk of cardio-cerebral vascular events: A meta-analysis of prospective cohort studies. Eur J Prev Cardiol. Published online 2017. doi:10.1177/2047487317702043

This article is protected by copyright. All rights reserved 
31. Bassetti CLA, Randerath W, Vignatelli L, et al. EAN/ERS/ESO/ESRS statement on the impact of sleep disorders on risk and outcome of stroke. Eur Respir J. Published online 2020. doi:10.1183/13993003.01104-2019

32. Hoevenaar-Blom MP, Spijkerman AMW, Kromhout D, Verschuren WMM. Sufficient sleep duration contributes to lower cardiovascular disease risk in addition to four traditional lifestyle factors: The MORGEN study. Eur J Prev Cardiol. Published online 2014. doi:10.1177/2047487313493057

33. Castro-Diehl C, Wood AC, Redline S, et al. Mediterranean diet pattern and sleep duration and insomnia symptoms in the Multi-Ethnic Study of Atherosclerosis. Sleep. Published online 2018. doi:10.1093/sleep/zsy158

34. Maire M, Linder S, Dvořák C, et al. Prevalence and management of chronic insomnia in Swiss primary care: Cross-sectional data from the "Sentinella" practice-based research network. J Sleep Res. Published online 2020. doi:10.1111/jsr.13121

35. Shochat T, Umphress J, Israel AG, Ancoli-Israel S. Insomnia in primary care patients. Sleep. Published online 1999.

36. Arroll B, Fernando A, Falloon K, Goodyear-Smith F, Samaranayake C, Warman G. Prevalence of causes of insomnia in primary care: A cross-sectional study. Br J Gen Pract. Published online 2012. doi:10.3399/bjgp12X625157

37. Bjorvatn B, Meland E, Flo E, Mildestvedt T. High prevalence of insomnia and hypnotic use in patients visiting their general practitioner. Fam Pract. Published online 2017. doi:10.1093/fampra/cmw107

38. Sivertsen B, Nordhus IH, Bjorvatn B, Pallesen SÅ. Sleep problems in general practice: A national survey of assessment and treatment routines of general practitioners in Norway. $J$ Sleep Res. Published online 2010. doi:10.1111/j.1365-2869.2009.00769.x

39. Everitt H, McDermott L, Leydon G, Yules H, Baldwin D, Little P. GPs' management strategies for patients with insomnia: A survey and qualitative interview study. Br J Gen Pract. Published online 2014. doi:10.3399/bjgp14X677176

40. Green LA, Fryer GE, Yawn BP, Lanier D, Dovey SM. The ecology of medical care revisited. $N$ Engl J Med. Published online 2001. doi:10.1056/NEJM200106283442611

41. Espie CA, Kyle SD, Hames P, Gardani M, Fleming L, Cape J. The Sleep Condition Indicator: A clinical screening tool to evaluate insomnia disorder. BMJ Open. Published online 2014.

This article is protected by copyright. All rights reserved 
doi:10.1136/bmjopen-2013-004183

42. Luik Al, Machado PF, Siriwardena N, Espie CA. Screening for insomnia in primary care: Using a two-item version of the Sleep Condition Indicator. Br J Gen Pract. Published online 2019. doi:10.3399/bjgp19X701045

43. Hammerschlag AR, Stringer S, De Leeuw CA, et al. Genome-wide association analysis of insomnia complaints identifies risk genes and genetic overlap with psychiatric and metabolic traits. Nat Genet. Published online 2017. doi:10.1038/ng.3888

44. Irish LA, Kline CE, Gunn HE, Buysse DJ, Hall MH. The role of sleep hygiene in promoting public health: A review of empirical evidence. Sleep Med Rev. Published online 2015. doi:10.1016/j.smrv.2014.10.001

45. Chung KF, Lee CT, Yeung WF, Chan MS, Chung EWY, Lin WL. Sleep hygiene education as a treatment of insomnia: A systematic review and meta-analysis. Fam Pract. Published online 2018. doi:10.1093/fampra/cm×122

46. Sateia MJ, Buysse DJ, Krystal AD, Neubauer DN, Heald JL. Clinical Practice Guideline for the Pharmacologic Treatment of Chronic Insomnia in Adults: An American Academy of Sleep Medicine Clinical Practice Guideline. J Clin Sleep Med. 2017;13(02):307-349. doi:10.5664/jcsm.6470

47. Saaresranta T, Hedner J, Bonsignore MR, et al. Clinical phenotypes and comorbidity in European sleep apnoea patients. PLoS One. Published online 2016. doi:10.1371/journal.pone.0163439

48. Janssen HCJP, Venekamp LN, Peeters GAM, Pijpers A, Pevernagie DAA. Management of insomnia in sleep disordered breathing. Eur Respir Rev. 2019;28(153):1-7. doi:10.1183/16000617.0080-2019

49. Anttalainen U, Grote L, Fietze I, et al. Insomnia symptoms combined with nocturnal hypoxia associate with cardiovascular comorbidity in the European sleep apnea cohort (ESADA). Sleep Breath. Published online 2019. doi:10.1007/s11325-018-1757-9

50. Agusti A. The path to personalised medicine in copd. Thorax. Published online 2014. doi:10.1136/thoraxjnl-2014-205507

51. Hohagen F, Kappler C, Schramm E, Riemann D, Berger M. Sleep Onset Insomnia, Sleep Maintaining Insomnia and Insomnia With Early Morning Awakening-Temporal Stability of Subtypes in a Longitudinal Study on General Practice Attenders. Sleep. 1994;17(May):551-

This article is protected by copyright. All rights reserved 
554. doi:10.1093/sleep/17.6.551

52. Edinger JD, Wyatt JK, Stepanski EJ, et al. Testing the reliability and validity of DSM-IV-TR and ICSD-2 insomnia diagnoses: Results of a multitrait-multimethod analysis. Arch Gen Psychiatry. Published online 2011. doi:10.1001/archgenpsychiatry.2011.64

53. Johann AF, Hertenstein E, Kyle SD, et al. Insomnia with objective short sleep duration is associated with longer duration of insomnia in the Freiburg Insomnia Cohort compared to insomnia with normal sleep duration, but not with hypertension. PLoS One. Published online 2017. doi:10.1371/journal.pone.0180339

54. Blanken TF, Benjamins JS, Borsboom D, et al. Insomnia disorder subtypes derived from life history and traits of affect and personality. The Lancet Psychiatry. Published online 2019. doi:10.1016/S2215-0366(18)30464-4

55. Jansen PR, Watanabe K, Stringer S, et al. Genome-wide analysis of insomnia in 1,331,010 individuals identifies new risk loci and functional pathways. Nat Genet. 2019;51(3):394-403. doi:10.1038/s41588-018-0333-3

56. Riemann D, Spiegelhalder K, Feige B, et al. The hyperarousal model of insomnia: A review of the concept and its evidence. Sleep Med Rev. Published online 2010. doi:10.1016/j.smrv.2009.04.002

57. Hertenstein E, Feige B, Gmeiner T, et al. Insomnia as a predictor of mental disorders: A systematic review and meta-analysis. Sleep Med Rev. Published online 2019. doi:10.1016/j.smrv.2018.10.006

58. Fortier-Brochu É, Beaulieu-Bonneau S, Ivers H, Morin CM. Insomnia and daytime cognitive performance: A meta-analysis. Sleep Med Rev. Published online 2012. doi:10.1016/j.smrv.2011.03.008

59. Orff HJ, Drummond SPA, Nowakowski S, Perlis ML. Discrepancy between subjective symptomatology and objective neuropsychological performance in insomnia. Sleep. Published online 2007. doi:10.1093/sleep/30.9.1205

60. Riedner BA, Goldstein MR, Plante DT, et al. Regional Patterns of Elevated Alpha and HighFrequency Electroencephalographic Activity during Nonrapid Eye Movement Sleep in Chronic Insomnia: A Pilot Study. Sleep. Published online 2016. doi:10.5665/sleep.5632

61. Siclari F, Tononi G. Local aspects of sleep and wakefulness. Curr Opin Neurobiol. Published online 2017. doi:10.1016/j.conb.2017.05.008

This article is protected by copyright. All rights reserved 
62. Krystal AD, Prather AA, Ashbrook LH. The assessment and management of insomnia: an update. World Psychiatry. 2019;18(3):337-352. doi:10.1002/wps.20674

63. Rios $\mathrm{P}$, Cardoso R, Morra D, et al. Comparative effectiveness and safety of pharmacological and non-pharmacological interventions for insomnia: an overview of reviews. Syst Rev. Published online 2019. doi:10.1186/s13643-019-1163-9

64. Beaulieu-Bonneau S, Ivers H, Guay B, Morin CM. Long-term maintenance of therapeutic gains associated with cognitive-behavioral therapy for insomnia delivered alone or combined with zolpidem. Sleep. Published online 2017. doi:10.1093/sleep/zsx002

65. Castronovo V, Galbiati A, Sforza M, et al. Long-term clinical effect of group cognitive behavioral therapy for insomnia: a case series study. Sleep Med. Published online 2018. doi:10.1016/j.sleep.2018.03.017

66. Cheung JMY, Jarrin DC, Ballot O, Bharwani A, Morin CM. A systematic review of cognitive behavioral therapy for insomnia implemented in primary care and community settings. Sleep Med Rev. Published online 2019. doi:10.1016/j.smrv.2018.11.001

67. Sweetman A, Lack L, Catcheside PG, et al. Cognitive and behavioral therapy for insomnia increases the use of continuous positive airway pressure therapy in obstructive sleep apnea participants with comorbid insomnia: A randomized clinical trial. Sleep. Published online 2019. doi:10.1093/sleep/zsz178

68. Longo DL, Soyka M. Treatment of benzodiazepine dependence. N Engl J Med. Published online 2017. doi:10.1056/NEJMra1611832

69. Parsaik AK, Mascarenhas SS, Khosh-Chashm D, et al. Mortality associated with anxiolytic and hypnotic drugs - A systematic review and meta-analysis. In: Australian and New Zealand Journal of Psychiatry. ; 2016. doi:10.1177/0004867415616695

70. Schonmann Y, Goren O, Bareket R, Comaneshter D, Cohen AD, Vinker S. Chronic hypnotic use at 10 years-does the brand matter? Eur J Clin Pharmacol. Published online 2018. doi:10.1007/s00228-018-2531-4

71. Fan X, Su Z, Nie S, et al. Efficacy and safety of Chinese herbal medicine Long Dan Xie Gan Tang in insomnia: A systematic review and meta-analysis. Medicine (Baltimore). Published online 2020. doi:10.1097/MD.0000000000019410

72. Mccleery J, Cohen DA, Sharpley AL. Pharmacotherapies for sleep disturbances in dementia. Cochrane Database Syst Rev. Published online 2016. doi:10.1002/14651858.CD009178.pub3

This article is protected by copyright. All rights reserved 
73. Morin $\mathrm{CM}$, Vallières $\mathrm{A}$, Guay $\mathrm{B}$, et al. Cognitive behavioral therapy, singly and combined with medication, for persistent insomnia: A randomized controlled trial. JAMA - J Am Med Assoc. Published online 2009. doi:10.1001/jama.2009.682

This article is protected by copyright. All rights reserved 